\section{Eosinophilic esophagitis with pseudodiverticulosis: a rare coincidence}

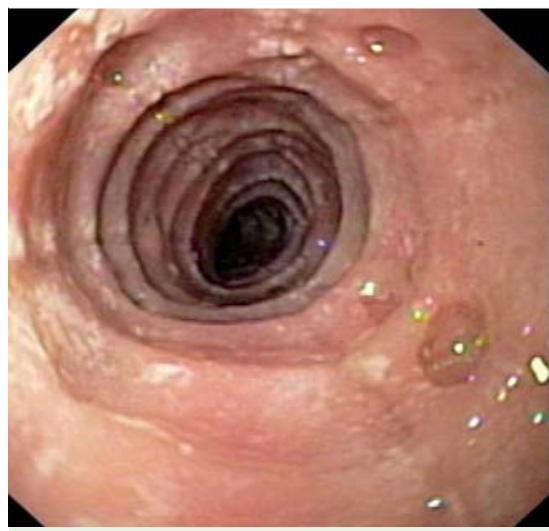

Fig. 1 Endoscopic view of the esophagus showing the concentric rings and multiple orifices of the pseudodiverticula.

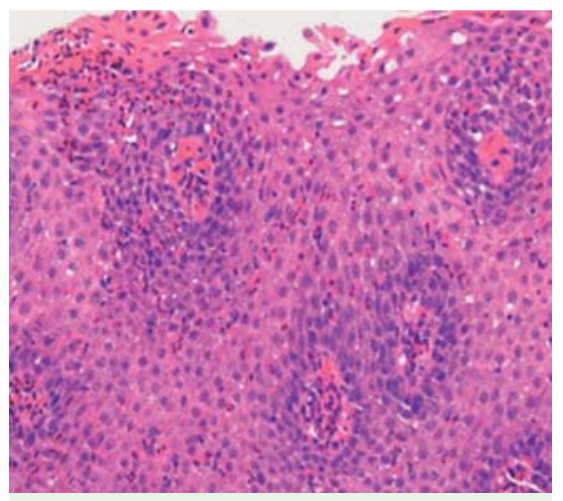

Fig. 2 Hematoxylin and eosin (H\&E)-stained esophageal mucosal biopsies showing a dense infiltrate of eosinophils (original magnification $\times 400)$.

We present a rare case of dysphagia due to the coincidence of eosinophilic esophagitis with esophageal intramural pseudodiverticulosis (EIP).

A 28-year-old man had a 15-year history of intermittent solid-food dysphagia and heartburn-like symptoms that were not relieved with prescription gastric acid-reducing agents. He denied any history of food impaction or allergies. Blood analysis and physical examination were unremarkable with a stable body mass index (BMI) of $21 \mathrm{~kg} / \mathrm{m}^{2}$.

A 24-hour esophageal $\mathrm{pH}$ study showed no evidence of pathological gastroesophageal reflux and esophageal manometry demonstrated no motor abnormalities. Upper gastrointestinal endoscopy showed a small caliber esophagus with multiple concen-

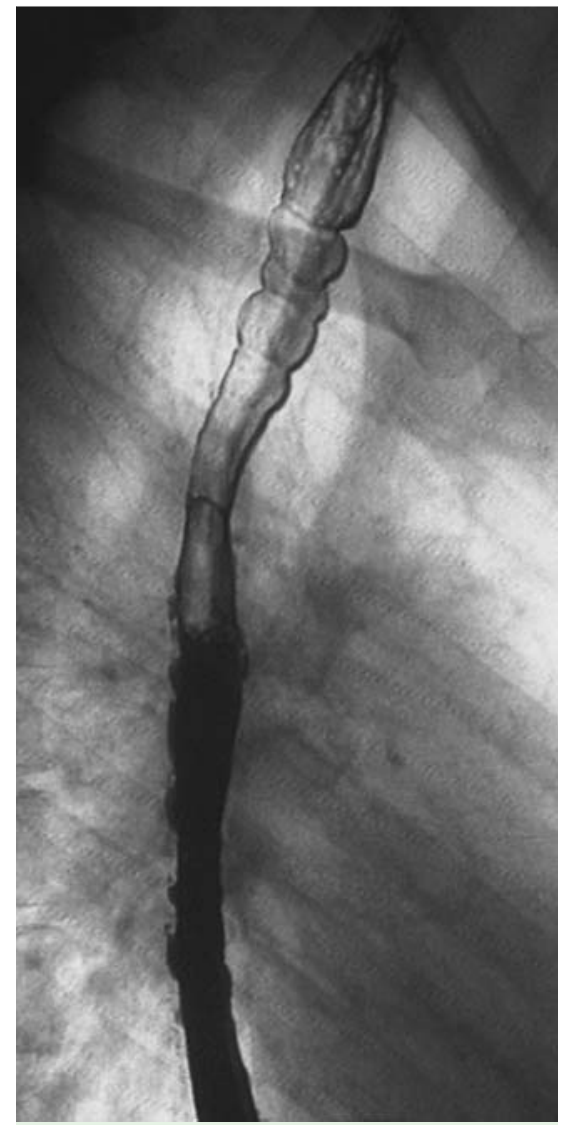

Fig.3 Image from the barium esophagram showing a small caliber esophagus with pseudodiverticula in the distal and mid-esophagus, and multiple rings in the proximal esophagus.

tric rings with additional white spots and geal biopsies revealed a high eosinophil count (>50 eosinophils/high power field; - Fig.2), indicative of eosinophilic esophagitis. A barium esophagogram also revealed a small caliber esophagus with tiny flask-shaped outpouchings in the distal and mid-esophagus and multiple rings in the proximal esophagus ( $\bullet$ Fig.3).

EIP is a rare entity, which often presents with dysphagia. Although its etiology is not precisely known, current data suggest that the pseudodiverticula represent dilated excretory ducts of mucosal glands. This dilatation may be caused either by long-term esophageal inflammation or by increased intra-esophageal pressure from altered motility or stenosis [1]. The coinmultiple small orifices ( $\mathbf{F i g}$. 1). Esopha- cidence of EIP with eosinophilic esophagitis is extremely rare with only two cases reported in the literature $[1,2]$. It is suspected that either chronic inflammation or stenosis may have led to the association with EIP in our patient.

Treatment of EIP is usually directed at the underlying associated condition [1]. The treatment of eosinophilic esophagitis is aimed at reducing eosinophilic inflammation with the use of corticosteroids. In addition, endoscopic dilation may be of benefit for esophageal strictures [3]. In our patient, a therapeutic regimen consisting of topical steroid intake led to significant clinical improvement within 4 weeks.

Endoscopy_UCTN_Code_CCL_1AB_2AC_3AH

Competing interests: None

\section{Müller ${ }^{1}$, R. C. Will ${ }^{2}$, A. J. Eckardt ${ }^{1}$}

${ }^{1}$ Department of Gastroenterology, German Diagnostic Clinic, Wiesbaden, Germany

2 Department of Gastroenterology, American Hospital Dubai, Dubai

\section{References}

1 Engel MA, Raithel M, Amann K et al. Rare coincidence of eosinophilic esophagitis with esophageal stenosis and intramural pseudodiverticulosis. Dig. Liver Dis 2008; 40: $700-$ 706

2 Tsai CM, Butler J, Cash BD. Pseudodiverticulosis with eosinophilic esophagitis: first reported case. Gastrointest Endosc 2007; 66: 1223-1224

3 Bohm M, Richter J. Treatment of eosinophilic esophagitis: overview, current limitations, and future direction. Am J Gastroenterol 2008; 103: $2635-2544$

\section{Bibliography}

Dol http://dx.doi.org/

10.1055/s-0031-1291588

Endoscopy 2012; 44: E71

(c) Georg Thieme Verlag KG

Stuttgart · New York

ISSN 0013-726X

\section{Corresponding author}

\section{Müller, MD}

Department of Gastroenterology

German Diagnostic Clinic

Aukammallee 33

D-65191 Wiesbaden

Germany

Fax: +49-611-577460

mueller.gastro@dkd-wiesbaden.de 